\title{
Quality assessment and control of high-throughput sequencing data
}

\author{
Mick Watson* \\ Edinburgh Genomics, The Roslin Institute, Edinburgh, UK \\ *Correspondence: mick.watson@roslin.ed.ac.uk
}

Edited and reviewed by:

Richard D. Emes, University of Nottingham, UK

Keywords: bioinformatics, genomics, transcriptomics, quality control, next-generation sequencing, sequencing, illumina

Second- and third-generation sequencing technologies are driving a revolution in biology and medicine, with ultra-high throughput sequencers now able to produce 200 human genomes every 3 days at a cost of $\$ 1000$ per genome (Watson, 2014). Meanwhile, in our lab at Edinburgh Genomics and in other labs throughout the World, researchers are generating their first single-molecule reads from a hand-held, USB-powered sequencer as part of Oxford Nanopore's MinION access programme (MinION Access Programme, 2014 ${ }^{1}$ ). Whilst the revolution in biology is recognized, the associated revolution in bioinformatics often goes unmentioned. This Frontiers "Research Topic" is about that revolution; it is about data, and data-driven discovery. The sequencers mentioned above, and others from Pacific Biosciences and Ion Torrent, produce either huge amounts of data, data that are very complex, or both. Bioinformaticians throughout the world are creating novel pipelines, algorithms and tools to be able to cope with the huge amount of diverse data types that can be produced. The very first step in many of those pipelines and tools is quality assessment, quality control and artifact removal. These issues all involve data-driven research-what can we learn from the data? What are the data telling us about quality and artifacts? The first group of papers in the research topic deal with quality assessment and reveal pipelines that are in use in sequencing facilities today. The second set of papers deal with applications of sequencing technologies to particular domains, and how we can improve those applications through effective control of quality and artifacts. The final set of papers deal with very specific biological questions, and what we can learn from the raw data to improve our analyses and help us to better answer those questions.

A series of bioinformatics pipelines are applied to sequencing data by the data generating facility, and it is important that those who work with sequencing data understand these. Leggett et al. (2013) reveal many of the pipelines and tools used at The Genome Analysis Centre (TGAC), a genomics institute based in Norwich, UK, which has access to every major sequencing platform. Their paper describes every step in the data generation pipeline, from their Laboratory Information Management System (LIMS) to data-specific pipelines for matepair and RAD-Seq libraries. Similarly, Paszkiewicz et al. (2014)

${ }^{1}$ https://www.nanoporetech.com/technology/the-minion-device-a-miniaturis ed-sensing-system/minion-access-programme describe bioinformatics quality-control pipelines used at the Exeter sequencing service, a smaller laboratory at the University of Exeter. Interestingly, they also include details of laboratory quality-control where relevant. Hadfield and Eldridge (2014) describe MGA, a tool that deals with two of the most frequently asked questions in DNA sequencing facilities: "how much did we sequence?" and "what did we sequence?" MGA presents a visual and tabular report to users detailing the yield for each lane in a sequencing run, as well as an assessment of the amount of adapter and contaminant DNA present. Finally, Trivedi et al. (2014) describe an important pipeline/method in use at Edinburgh Genomics, a genomics facility at the University of Edinburgh. Many QC metrics involve mapping to a reference genome, and Trivedi et al ask a simple question: what can we learn when there is no reference genome? Their paper deals with assessment of insert size, duplication rate and contamination by first generating a simple and fast de novo assembly of the data, and they show that even when the assembly is poor, accurate QC metrics can be generated.

The second group of papers deals with the application of quality control to specific types of sequencing library. Macmanes (2014) looks at the effect of quality trimming on mRNA sequencing data, and specifically assembly of those data. Quality trimming is ubiquitous in many bioinformatics pipelines, with many choosing an arbitrary Q-score of 20 or 30 as a cut-off. However, MacManes shows that such "aggressive" trimming can have a large effect on the results, and suggests a much lower Q-score cut-off if the purpose of the experiment is transcript discovery. Mbandi et al. (2014) tackle exactly the same problem, and come to similar conclusions, showing that transcript assemblies from untrimmed data result in better alignments and more contiguous assemblies. Finally, Turner (2014) describes a bioinformatics protocol for assessing insert size and adapter content in Nextera XT libraries. The Nextera XT protocol uses transposomes to fragment DNA and is very sensitive to the concentration of input DNA. These libraries can result in fragments that are far smaller than the read length, and Turner describes a protocol that can cope with this problem.

The final group of papers deal with quality and artifact assessment applied to specific biological problems. Amaral et al. (2014) describe the problem of RNA-Seq in small invertebrates. As the traditional RNA-Seq protocol requires a relatively large amount of RNA as input, invertebrates such as Drosophila need to be pooled prior to sequencing. This pooling can produce 
additional complexity in the data, and Amaral et al. describe the use of marker genes to identify data points that suffer from contamination and should be removed from further analysis. Complementary to Macmanes (2014), Mbandi et al. (2014) and Amaral et al. (2014) find that trimming of the $5^{\prime}$ end, to remove biases introduced by random hexamer priming (Hansen et al., 2010), improves the mapping rate. Carroll et al. (2014) deal with ChIP-Seq and ChIP-exo data and suggest that standard metrics for assessing the quality of these data types should be assessed at every step of data pre-processing. They also suggest that ChIPexo data requires different processing steps and an adaptation of cross-correlation metrics. Finally, Kumar et al. (2013) describe a method for discovery and removal of "contaminants," but here the term is extended to include symbionts and parasites. They show that "Blobology," separation of assembled contigs using GC content and coverage, can be used to bin data into distinct groups that can then be assembled separately, resulting in better assemblies and useful data from organisms that may be difficult to study in isolation.

The papers in this Frontiers Research Topic represent a diverse and fascinating collection, and should be of interest to a wide audience, including bioinformaticians keen to improve their pipelines and biologists keen to learn more about the complexity of second- and third-generation sequencing data.

\section{ACKNOWLEDGMENT}

Mick Watson receives funding from the UK Biotechnology and Biological Sciences Research Council (BBSRC) (BB/J004243/1).

\section{REFERENCES}

Amaral, A. J., Brito, F. F., Chobanyan, T., Yoshikawa, S., Yokokura, T., Van Vactor, D., et al. (2014). Quality assessment and control of tissue specific RNAseq libraries of Drosophila transgenic RNAi models. Front. Genet. 5:43. doi: 10.3389/fgene. 2014.00043

Carroll, T. S., Liang, Z., Salama, R., Stark, R., and De Santiago, I. (2014). Impact of artifact removal on ChIP quality metrics in ChIP-seq and ChIP-exo data. Front. Genet. 5:75. doi: 10.3389/fgene.2014.00075
Hadfield, J., and Eldridge, M. D. (2014). Multi-genome alignment for quality control and contamination screening of next-generation sequencing data. Front. Genet. 5:31. doi: 10.3389/fgene.2014.00031

Hansen, K. D., Brenner, S. E., and Dudoit, S. (2010). Biases in Illumina transcriptome sequencing caused by random hexamer priming. Nucleic Acids Res. 38, e131. doi: 10.1093/nar/gkq224

Kumar, S., Jones, M., Koutsovoulos, G., Clarke, M., and Blaxter, M. (2013). Blobology: exploring raw genome data for contaminants, symbionts and parasites using taxon-annotated GC-coverage plots. Front. Genet. 4:237. doi: 10.3389/fgene.2013.00237

Leggett, R. M., Ramirez-Gonzalez, R. H., Clavijo, B. J., Waite, D., and Davey, R. P. (2013). Sequencing quality assessment tools to enable data-driven informatics for high throughput genomics. Front. Genet. 4:288. doi: 10.3389/fgene.2013. 00288

Macmanes, M. D. (2014). On the optimal trimming of high-throughput mRNA sequence data. Front. Genet. 5:13. doi: 10.3389/fgene.2014.00013

Mbandi, S. K., Hesse, U., Rees, D. J., and Christoffels, A. (2014). A glance at quality score: implication for de novo transcriptome reconstruction of Illumina reads. Front. Genet. 5:17. doi: 10.3389/fgene.2014.00017

Paszkiewicz, K. H., Farbos, A., O'neill, P., and Moore, K. (2014). Quality control on the frontier. Front. Genet. 5:157. doi: 10.3389/fgene.2014.00157

Trivedi, U. H., Cezard, T., Bridgett, S., Montazam, A., Nichols, J., Blaxter, M., et al. (2014). Quality control of next-generation sequencing data without a reference. Front. Genet. 5:111. doi: 10.3389/fgene.2014.00111

Turner, F. S. (2014). Assessment of insert sizes and adapter content in fastq data from NexteraXT libraries. Front. Genet. 5:5. doi: 10.3389/fgene.2014.00005

Watson, M. (2014). Illuminating the future of DNA sequencing. Genome Biol. 15, 108. doi: $10.1186 / \mathrm{gb} 4165$

Conflict of Interest Statement: The author declares that the research was conducted in the absence of any commercial or financial relationships that could be construed as a potential conflict of interest.

Received: 02 July 2014; accepted: 02 July 2014; published online: 25 July 2014. Citation: Watson M (2014) Quality assessment and control of high-throughput sequencing data. Front. Genet. 5:235. doi: 10.3389/fgene.2014.00235

This article was submitted to Bioinformatics and Computational Biology, a section of the journal Frontiers in Genetics.

Copyright (C) 2014 Watson. This is an open-access article distributed under the terms of the Creative Commons Attribution License (CC BY). The use, distribution or reproduction in other forums is permitted, provided the original author(s) or licensor are credited and that the original publication in this journal is cited, in accordance with accepted academic practice. No use, distribution or reproduction is permitted which does not comply with these terms. 